\title{
Resistance Training
}

National Cancer Institute

\section{Source}

National Cancer Institute. Resistance Training. NCI Thesaurus. Code C154219.

Physical exercise that uses weight, or other forms of resistance, to induce muscle

contraction and build strength, anaerobic endurance, and size of skeletal muscles. Typical

exercises include leg and chest press, knee extension, leg curl, row, abdominal crunch,

and bicep curl. 